\title{
Norois
}

Environnement, aménagement, société

\section{Voie express et ségrégation. Les fluctuations du marché foncier et immobilier aux franges ouest de l'île-de-France}

Expressway and segregation. The fluctuations of land and property market on the urban fringe in the west of the region Île-de-France

\section{Claire Aragau}

\section{OpenEdition}

Journals

Édition électronique

URL : http://journals.openedition.org/norois/1276

DOI : 10.4000/norois. 1276

ISBN : 978-2-7535-1553-6

ISSN : $1760-8546$

Éditeur

Presses universitaires de Rennes

\section{Édition imprimée}

Date de publication : 1 décembre 2007

Pagination : 81-94

ISBN : 978-2-7535-0616-9

ISSN : 0029-182X

Référence électronique

Claire Aragau, "Voie express et ségrégation. Les fluctuations du marché foncier et immobilier aux franges ouest de l'île-de-France », Norois [En ligne], 205 | 2007/4, mis en ligne le 01 décembre 2009, consulté le 01 mai 2019. URL : http://journals.openedition.org/norois/1276 ; DOI : 10.4000/ norois. 1276 


\title{
VOIE EXPRESS ET SÉGRÉGATION \\ LES FLUCTUATIONS DU MARCHÉ FONCIER ET IMMOBILIER AUX FRANGES OUEST DE L'ÎLE-DE-FRANCE
}

\author{
Claire Aragau \\ GECKO \\ (Université Paris 10), \\ 200 avenue de la République - 92001 NANTERRE cedex \\ claire.aragan@free.fr
}

\section{RÉSUMÉ}

Dans un contexte de hausse des prix de l'immobilier et du foncier amorcée dès 1998, la nouvelle N12, devenue voie express entre Bois-d'Arcy et Dreux, se présente comme une voie d'accès à une réserve foncière et à des biens immobiliers abordables financièrement pour certaines catégories de ménages. Mais rapidement, avec la valorisation de l'espace, cette réserve devient plus sélective.

La N12 dessert des communes de la banlieue ouest de Paris et en particulier situées dans le département des Yvelines, connu pour la préservation de ses paysages. Une fois la nouvelle route aménagée les réactions de défense de l'environnement se font plus vives. D'une simple hausse des prix, il se pourrait que l'on passe à une véritable pression foncière et immobilière. Qu'en est-il réellement? La déconnexion entre les prix fonciers et les prix immobiliers fait apparaître une pression immobilière atténuée par rapport à une pression foncière plus marquée. Celle-ci s'exerce dans les communes en fonction de leur profil avant l'aménagement de l'infrastructure et non en fonction de leur localisation sur l'axe. Le passage de la voie express ou sa proximité ne joue qu'une fois la valorisation entamée, c'est-à-dire qu'elle ne fait que l'accentuer.

\section{Mots CLÉ : Périurbain - Pression foncière - Pression immobilière - Voie express.}

\section{ABSTRACT}

\section{Expressway and segregation. The fuctuations of land and property market} on the urban fringe in the west of the region Île-de-France

In a context of price rise on land and property market from the year 1998 on, the new N12, which has become an expressway between Bois d'Arcy and Dreux, appears as an access road to a land reserve and real estate affordable for a certain category of households. However this reserve is becoming more selective because of the rise in value of the area.

The N12 serves municipalities of the western suburb of Paris and especially those located in the Yvelines department, known for the preservation of its landscapes. The building of the new expressway triggers off stronger reactions of preservation of the environment. This could lead from a simple price increase to a real pressure on the land and the property market. What is really happening? The disconnection between the price of land and the price of real estate shows a slight pressure on the property market in comparison to a 
stronger pressure on the land market. The latter is exerted on municipalities depending on their profiles before the building of the infrastructure and not to their location on the express way. The building of the expressway through the municipalities or next to them plays an important role only after the area has begun to rise in value, this means it just enhances the prices.

KEY WORDS : Express Way-Peri-urban Area-Pressure on the land market-Pressure on the property market

Selon les dernières analyses recueillies dans l'Atlas rural et agricole de l'T̂le-de-France (De Biasi et Ordonnet, 2004, p. 46), la vie à la campagne séduit beaucoup de Français : 84 \% d'entre eux estiment que la vie est plus agréable à la campagne qu'en ville et « les Franciliens partagent largement cette opinion. S'ils pouvaient choisir sans aucune contrainte, plus de la moitié des ruraux franciliens ${ }^{1}$ souhaiteraient rester dans l'espace rural francilien ». Après la qualité de vie (45\%), les ruraux d'Île-de-France considèrent que c'est l'accès aux équipements et aux services qui caractérise le mieux leur cadre de vie : être ni trop près de la ville, ni trop loin (28\%). La tendance générale est à vouloir bénéficier à la fois de la qualité de vie que réserve la campagne et de la proximité de la ville pour le travail, les services, les loisirs (Dubois-Taine et Chalas, 1997). L'espace rural dont il est question est à chaque fois situé à la périphérie d'une ville; il ne fait que recouvrir les caractéristiques du périurbain. Ce lieu de résidence se distingue par l'absence de sentiment d'isolement.

Les aménagements réalisés récemment sur la N12, qui l'ont fait accéder au statut de voie express entre Bois d'Arcy et Dreux, permettent au territoire qu'elle dessert de bénéficier à la fois d'une meilleure accessibilité à un pôle urbain et d'un environnement préservé. Se pose alors la question de leurs effets sur l'évolution du marché immobilier et foncier, qu'il convient d'aborder avec une grande prudence méthodologique. Comme le rappelle F. Plassard « la vision simpliste de mécanismes de cause à effet ne peut être conservée dès qu'on étudie les relations entre autoroute et développement régional» (Offner, 1993). Des interprétations erronées proviennent par exemple d'une comparaison hâtive entre un "avant » et un "après ». De plus, si une "étude d'impact » peut être faite sur le milieu naturel, il en va autrement pour l'environnement socio-économique. Il faut envisager un diagnostic « en dynamique », c'est-à-dire que l'étude ne doit pas se faire de façon linéaire mais elle doit mettre en interaction des événements. Comment comparer deux périodes qui ne peuvent pas l'être tant le contexte politique, économique et social a évolué? Ponctuellement, l'analyse diachronique est envisageable; mais elle ne peut suffire. En choisissant comme angle d'approche la voie express, nous tenterons de montrer comment elle entre en interaction avec d'autres phénomènes, notamment la stratégie d'acteurs tels que des professionnels de l'immobilier et du foncier mais aussi les particuliers, pour comprendre les fonctionnements du marché et leurs répercussions socio-spatiales.

Si les nouvelles infrastructures de transport réalisées dans le périurbain ont des incidences sur le marché immobilier et foncier, celles-ci sont de natures différentes. En premier lieu, dans le contexte de hausse des prix de l'immobilier et du foncier amorcée dès 1998, un axe de communication aux performances améliorées peut être considéré comme une voie d'accès à une réserve

1. Commentaire des auteurs de l'Atlas rural et agricole d'Île-de-France, (De Biasi et Ordonnet, 2004) : «Alors que l'Ile-deFrance accueille l'une des plus grosses agglomérations urbaines européennes, une part importante des franciliens conserve le sentiment d'habiter encore à la campagne. Le territoire d'Ile-de-France demeure composé à près de $80 \%$ d'espaces ruraux, agricoles ou forestiers. La notion de rural englobe celles de campagne et de nature. C'est sur cette notion forte de territoire, plutôt que sur les modes de vie et les migrations alternantes, que s'est fondée la délimitation urbain/rural utilisée dans cet atlas. Après une période récente marquée par le productivisme et l'étalement urbain, l'espace rural francilien reste fragile et l'une des principales motivations de cet atlas est de faire progresser une « discrimination positive » de la ruralité. » 
foncière et à des biens immobiliers abordables financièrement pour certaines catégories de ménages. Mais dans un second temps, cette réserve risque de faire l'objet d'une pression immobilière et foncière et de devenir inaccessible aux moins aisés, ce qui enclenche un processus de ségrégation socio-spatiale. Celui-ci sera examiné au regard des transformations effectuées sur la N12 devenue voie express entre Bois-d'Arcy et Dreux ${ }^{2}$.

\section{Accéder à une offre meilleur marché}

\section{LA RÉSERVE IMMOBILIÈRE ET FONCIÈRE AUX ABORDS DE LA N12 : PRÉCISÉMENT OÙ ET POUR QUI?}

En raison des performances techniques accrues de l'axe routier, les distances temps sont réduites mais aussi garanties par la fluidité de la circulation (absence de carrefours à niveau, interdiction aux véhicules lents) et une limite supérieure de vitesse portée à $110 \mathrm{~km} / \mathrm{h}$. D'après l'enquête menée par la DDE préalablement à la $\mathrm{DUP}^{3}$ de 1992, le gain de temps représenterait six minutes pour les véhicules légers et huit minutes pour les poids lourds. Le gain est plus important à l'endroit d'anciens points névralgiques, comme la traversée de Jouars-Pontchartrain. Le nombre d'échangeurs, un tous les deux à trois kilomètres, permet des connexions fréquentes avec les voies transversales. La RN12 est incontestablement l'axe structurant le réseau routier. C'est autour de cette épine dorsale que s'articule le système viaire, incluant les routes nationales, départementales et communales.

En se penchant sur l'évolution des transactions, il est possible de mieux saisir la manne que représente ce secteur de la N12 en terme d'accessibilité immobilière et foncière. La source qui permet de connaître ces volumes est la base BIEN, alimentée par la chambre interdépartementale des notaires parisiens ${ }^{4}$. L'année de départ choisie est l'année 1998 car c'est à partir de cette date que le taux de couverture devient satisfaisant pour les communes de la grande couronne. Comme c'est en février 2001 que la N12 acquiert le statut de voie express avec l'ouverture de la déviation de Jouars-Pontchartrain, s'arrêter en 2003 permet de disposer de deux périodes de comparaison comprenant le même nombre d'années, c'est-à-dire trois, avant et après les travaux d'aménagement. Cette démarche diachronique s'inscrit dans un cadre systémique plus large pour proposer des facteurs d'explications aux observations faites.

Concernant les transactions de maisons individuelles, les volumes ne cessent d'augmenter jusqu'en 2002 pour ensuite amorcer une baisse à partir de 2003 (fig. 1). Plusieurs explications interviennent. Tout d'abord, le contexte national est à prendre en compte. La concomitance entre l'envolée des prix, la baisse des droits de mutation, des conditions d'emprunt exceptionnelles, a entrâné une hausse des transactions. L'évolution des prix de l'immobilier à l'achat mais aussi celle des loyers n'offrent que peu d'alternatives à une part sans cesse plus élevée de ménages : c'est

2. Ce travail a été réalisé dans le cadre d'une thèse de doctorat sous la direction de J.-P. Charvet (Aragau, 2007). L'étude porte sur 96 communes, 55 d'entre elles se trouvent dans les Yvelines et 41 en Eure-et-Loir. La population est passée de 110267 habitants en 1990 à 123656 en 1999. Les deux tiers des communes ont moins de 1000 habitants et un tiers d'entre elles en ont moins de 400.

3. Une Déclaration d'Utilité Publique est une procédure administrative qui permet de réaliser une opération d'aménagement, comme la création d'une infrastructure de communication par exemple, sur des terrains privés en les expropriant, précisément pour cause d'utilité publique; elle est obtenue à l'issue d'une enquête d'utilité publique.

4. Les données de la base BIEN (Base d'Informations Économiques Notariales) couvrent Paris et la petite couronne à partir de 1990 et la grande couronne depuis 1996. Le taux de couverture avoisine les $80 \%$ sur les cinq dernières années pour les Yvelines. Le taux de couverture représente le rapport entre le nombre d'observations traitées sur la base CD-BIEN et le nombre estimé de ventes. Cette base ne traitant pas l'exhaustivité des actes enregistrés par les offices notariaux en France, celle-ci est « redressée » à partir du dénombrement des actes inscrits au répertoire des offices d'Île-de-France. L'Observatoire régional du foncier en Île-de-France dispose de ces données pour le département des Yvelines mais pas de celles concernant l'Eure-et-Loir enregistrées dans la base Perval. Pour ce dernier département, notre étude des marges de l'Île-de-France a donc été réalisée de façon moins exhaustive, à partir du site de Particulier à Particulier, de données ponctuelles fournies par des notaires ainsi que par une enquête auprès des ménages. Les sources étant différentes pour les Yvelines et l'Eure-et-Loir les cartes réalisées ne prendront en compte que les chiffres issus de la base Bien. 


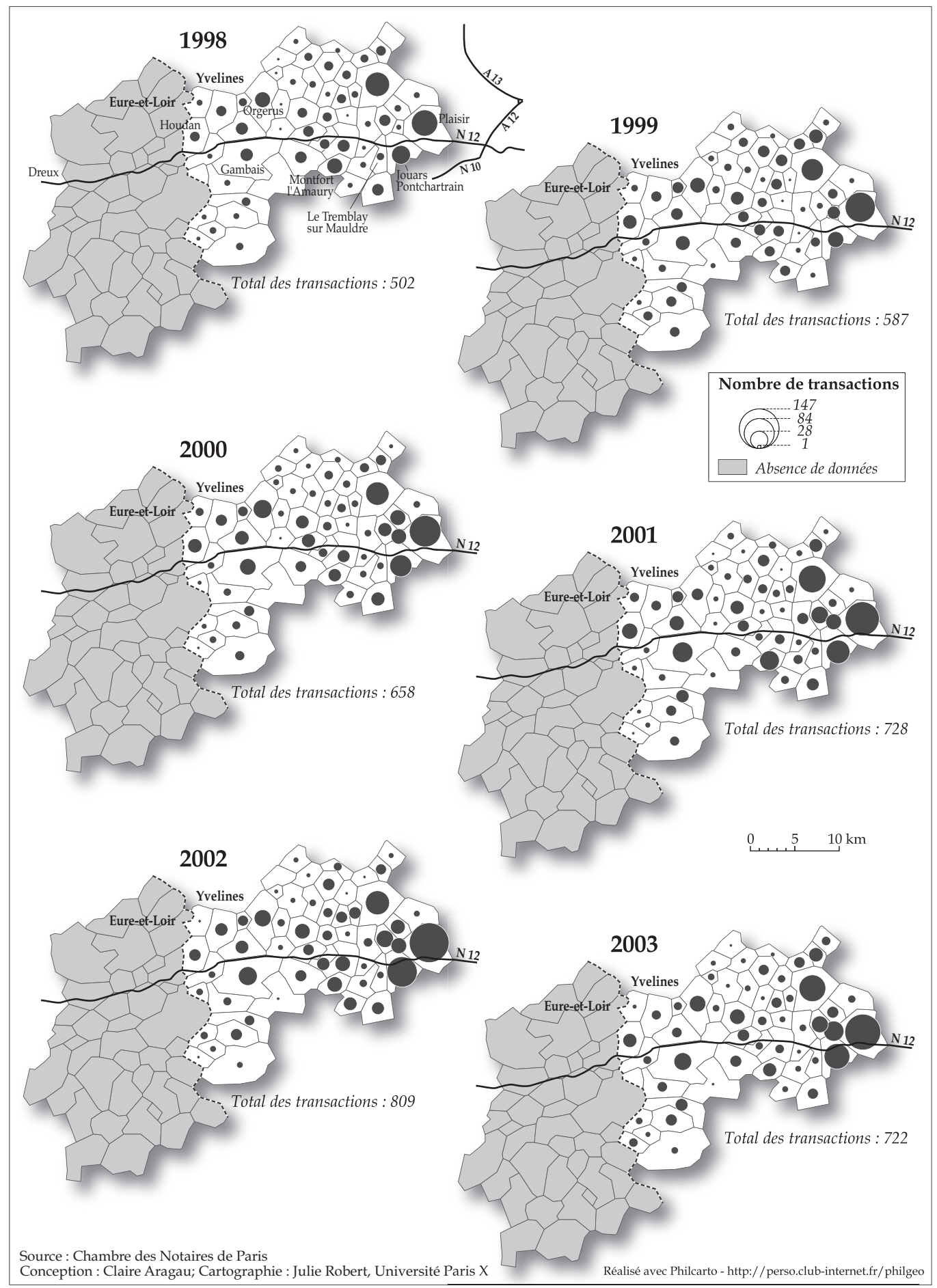

Figure 1 : Transactions de maison le long de la N12 - volume des ventes entre 1998 et 2003

Transactions of houses next to the N12 - The volume of sales between 1998 and 2003 
« soit l'abandon de la ville, la réalisation d'un projet résidentiel de plus en plus loin des centres urbains convoités..., soit la résignation à accepter un habitat de piètre qualité et de confort réduit » (Mouillart, 2006). Habiter non loin de la N12 relève de cette première possibilité. Les causes de l'emballement des prix sont justement « les conditions de crédit remarquables : le niveau des taux, la durée des prêts accordés, la diversité de l'offre et des produits... » (Mouillart, 2006). Les anciens propriétaires ont pu revendre en cherchant à réaliser une plus-value tandis que les nouveaux acquéreurs ont fait de même en revendant plus cher un bien qui leur a permis d'accéder à un logement plus spacieux situé à proximité de la N12 (Berger, 1993). La hausse des prix semble avoir pesé sur les capacités d'expansion du marché, ce qui expliquerait le recul observé en 2003. À partir de cette date, la structure des flux de l'accession se fait au bénéfice des ménages aux revenus élevés et parallèlement au détriment des primo-accédants. Comme le souligne M. Mouillart (2006), « le risque d'une telle situation est alors celui du renforcement massif des mécanismes de ségrégation et d'exclusion qui sont déjà à l'œuvre derrière cette progression des prix de l'immobilier résidentiel ». Cette tendance s'observe dans l'ensemble de l'Île-de-France mais le passage de la voie express fait intervenir des spécificités liées à la valorisation du secteur.

Dans la première période (1998-2000), les volumes augmentent dans des communes situées non loin de la N12 mais aussi et surtout dans celles qui se trouvent au nord de l'axe routier, tandis qu'au sud le nombre de transactions semble constant. Les communes situées au nord ont des profils socioprofessionnels moins qualifiés qu'au sud et la cote des maisons y est également moins élevée. Les propriétaires de pavillons dans des communes situées au nord peuvent ainsi espérer profiter d'une hausse plus spectaculaire de leur bien puisqu'ils partent de plus bas. L'anticipation de l'aménagement de la N12 en voie express, accentue ce phénomène de valorisation. Après 2001, l'effet de proximité se renforce et le marché profite aux catégories les plus aisées. Ainsi les communes les plus proches de Paris et les mieux situées le long de l'axe conservent les plus gros volumes de vente. À partir de cette date, la partie nord de la zone connaît un ralentissement des transactions, ce qui correspond aux limites des capacités d'expansion du marché tandis qu'au sud et à l'est les transactions se poursuivent dans les communes les mieux cotées.

Ces observations sont à mettre en relation avec les transactions sur les terrains à bâtir, qui permettent de compléter l'analyse. En effet le prix du terrain peut directement rendre compte de la valorisation d'un lieu. Celle-ci est plus difficilement perceptible à partir des transactions portant sur des maisons car elle est masquée par des différences concernant le nombre de pièces ou la qualité de la construction du logement. De plus, à travers l'augmentation importante du volume et des prix des ventes de terrains ou au contraire leur décélération soudaine, il est possible d'appréhender les stratégies d'anticipation des promoteurs et des élus. Ainsi entre 1998 et 2003 le volume des transactions de terrain à bâtir est très variable (fig. 2) et répond à d'autres logiques que celles des ventes de maisons déjà construites. Il est souvent lié à des projets lancés par des promoteurs ou, dans notre secteur d'étude, plutôt par des lotisseurs. Les lieux et les modalités d'intervention de professionnels de l'immobilier et du foncier apportent des éléments d'explication pour comprendre les variations locales du marché. Un promoteur fait lui-même la demande de permis de construire pour les maisons qu'il bâtit, tandis qu'un lotisseur prépare des lots et ce sont les particuliers qui déposent individuellement une demande de permis. Ils font ensuite appel au constructeur de leur choix. La baisse des transactions peut ainsi correspondre à la fin d'un programme. Les promoteurs et les lotisseurs se portent là où se trouvent la demande et les ménages solvables. Leur stratégie est également de lancer des opérations là où les prix ne pourront qu'augmenter par la suite. Ainsi cette catégorie d'acteurs est connue pour ses capacités d'anticipation. Dès lors, on constate, tout comme pour les maisons, une plus grande importance des transactions au nord de la voie express. La question qui se pose est de savoir si ces terrains sont promis à une valorisation plus forte que ceux situés au sud, déjà très coûteux, ou si, par ses nuisances, la route n'a pas eu l'effet inverse, c'est-à-dire la dévalorisation d'un espace et donc une augmentation des ventes liée à des prix bradés. Mis à part le coteau de Chennevières, secteur très résidentiel situé dans la commune de Jouars-Pontchartrain et entaillé par la route, la voie express est relativement éloignée des zones 


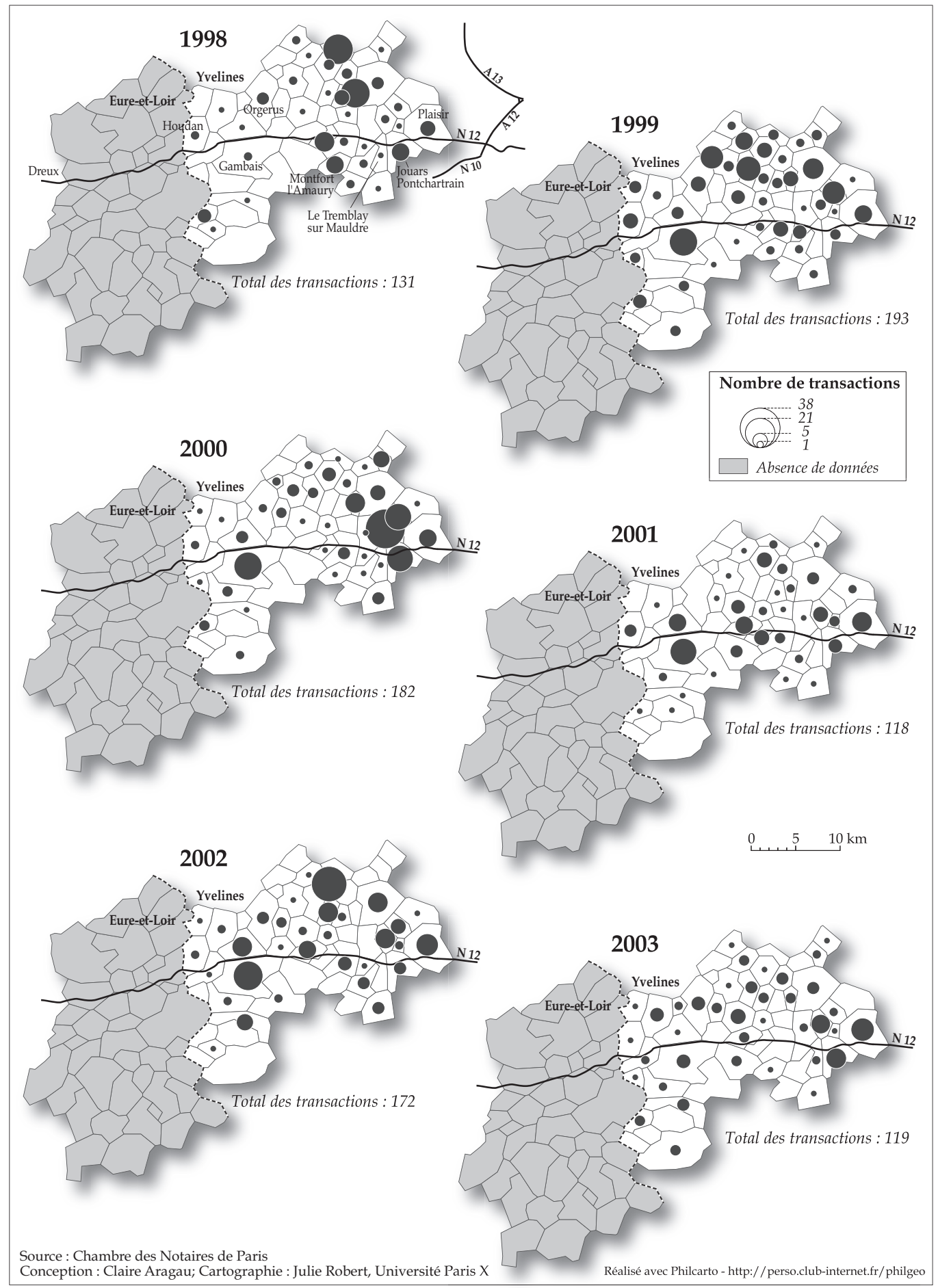

Figure 2 : Transactions des terrains le long de la N12 - volume des ventes entre 1998 et 2003

Transactions of lands next to the N12 - The volume of sales between 1998 and 2003 
bâties et présente des contraintes moindres par rapport à l'ancienne route nationale. Les résidents les plus proches de la voie express affirment que la continuité du trafic a réduit largement les nuisances sonores : les feux de signalisation sur l'ancienne voie entraînaient des arrêts et redémarrages des véhicules, notamment des poids lourds, particulièrement gênants.

L'année 2001 semble marquer un seuil : des opérations se terminent, les volumes des ventes affichent un recul. Les projets d'anticipation liés au passage de la voie express sont réalisés. Après cette année charnière, les transactions sont plus concentrées dans les communes proches de l'axe de la N12. La déviation de Jouars-Pontchartrain est ouverte. La valorisation des terrains est effective. Les ventes s'adressent alors à des catégories sociales plus aisées, ce qui limite l'expansion du marché. Une autre explication intervient pour comprendre cette baisse du volume des ventes : c'est la réaction des collectivités locales au passage de la voie express. La crainte d'une déferlante urbaine, dans un espace connu pour la qualité de son environnement, a provoqué une limitation volontaire des disponibilités foncières de la part des communes.

\section{UNE ACCESSION SELECTIVE}

La voie express permet donc l'accès à un bien immobilier pour des ménages qui, avec la valorisation de l'espace traversé, appartiennent à des catégories sociales de plus en plus aisées. La réserve foncière est bien réelle mais devient plus sélective : la ségrégation socio spatiale s'accentue. Derrière la recherche d'un logement plus spacieux pour un budget donné, se cachent d'autres critères de choix pour venir habiter ces communes proches de la N12. M. Mouillart résume la situation de la façon suivante : « Tout se passe comme si, afin de préserver les espaces qu'ils se sont constitués, les ménages aux revenus moyens et élevés acceptaient de payer un ticket d’entrée sur chacun des marchés qu'ils convoitent : plus le prix payé est élevé, moins le risque de devoir côtoyer des populations indésirables est fort. C'est la fameuse théorie des clubs à laquelle les économistes se réfèrent plus ou moins implicitement depuis une trentaine d'années lorsqu'ils analysent la formation des exclusions dans le secteur du logement : le marché est naturellement producteur d'exclusion et la demande en est demanderesse! Alors elle paye afin d'obtenir ce qu'elle souhaite et ceux qui ne peuvent suivre vont habiter ailleurs...» (Mouillart, 2006). Une enquête réalisée auprès des ménages ${ }^{5}$ permet de saisir leurs autres motivations.

Parmi les nouveaux installés dans la zone, ceux qui sont arrivés dans la commune il y a moins de dix ans, la motivation première est l'accès à un foncier et à un immobilier meilleur marché. La meilleure qualité de vie arrive immédiatement en seconde position. C'est seulement pour les personnes installées depuis plus de vingt ans que le critère «être proche du lieu de travail ou de celui du (de la) conjoint(e) » apparaît souvent.

Les éléments appréciés du lieu de résidence sont d'abord la dimension du logement, tout particulièrement pour les nouveaux résidents quel que soit le département d'installation, l'Eure-et-Loir ou les Yvelines, puis viennent le calme et l'environnement paysager. Le critère environnement social est rarement cité. Il est quelquefois un élément de choix dans des communes des Yvelines et très peu mentionné en Eure-et-Loir. Ce critère est certainement difficilement avouable. C'est pourtant dans le département des Yvelines que la ségrégation sociale est la plus nette sur notre terrain d'étude (Charmes, 2005). La notion d'environnement social est peut-être liée au critère de calme dans l'esprit des personnes interrogées. Dans les Yvelines, ces termes sont retenus à propos de l'environnement social des établissements scolaires publics. C'est un élément bien connu qui intervient dans le choix du lieu de résidence pour les familles ayant de jeunes enfants. $90 \%$ des

5. Une enquête a été réalisée auprès de ménages résidant dans quatre communes proches de la voie express, dans les Yvelines et en Eure-et-Loir. Le nombre d'enquêtés est de 30 ménages dans chacun des deux départements. L'échantillon se compose de : 1) Dix ménages récemment installés sur la commune (depuis moins de dix ans);2) Dix ménages installés depuis plus de dix ans ; 3) Dix ménages de retraités disposant d'un véhicule quelle que soit la date d'emménagement sur la commune. Le niveau de satisfaction des ménages, quant à leur commune de résidence, est mesuré sur une échelle comprenant 5 paliers : Pas du tout satisfait, Peu satisfait, Moyennement satisfait, Assez satisfait, Très satisfait. 
personnes interrogées se déclarent « assez satisfaites » et « très satisfaites » de leur lieu de résidence. Aucun enquêté ne se déclare «pas du tout satisfait» ou «moyennement satisfait». Ceci montre bien que la volonté d'habiter la commune résulte de la décision d'améliorer sa qualité de vie (Jaillet et al., 2005). Ce n'est pas une décision subie, contrairement à ce qui se produit dans de nombreuses communes de banlieue des villes du secteur. Évidemment ce choix reste guidé par le marché foncier et immobilier. La N12 permet donc l'accès à un secteur privilégié sans entraîner des nuisances fortes.

\section{D'une hausse des prix à une pression immobilière et foncière}

\section{UNE RÉALITÉ CONTRASTÉE}

Une fois la nouvelle route aménagée, les réactions de défense de l'environnement se font plus vives. Afin de protéger l'une des dernières entrées vertes de l'agglomération parisienne, les associations et de très nombreux élus réclament, le classement de la plaine de Jouars au titre de la loi du 2 mai 1930 relative à la protection des sites naturels. La réserve foncière et immobilière évoquée précédemment risque fortement d'être remise en cause. La crainte d'une urbanisation de la région peut laisser penser que les disponibilités foncières iront en s'amenuisant et par conséquent que le marché immobilier sera vite saturé. D’une simple hausse des prix, on pourrait passer à une véritable pression foncière et immobilière. Qu'en est-il réellement? Le contexte régional est-il propice à cette situation?

On observe effectivement une hausse des prix de l'immobilier. Pour la mesurer, on a séparé les transactions sur les maisons et celles portant sur les terrains. Un prix au $\mathrm{m}^{2}$ pour une maison ne signifie pas grand-chose, puisque le prix global d'une maison dépend de nombreux facteurs : de la situation, mais aussi de la qualité de la construction, du nombre de pièces. Raisonner à partir de fourchettes de prix permet de connaître immédiatement la valeur des biens vendus et leur proportion par rapport à d'autres. Ces fourchettes de prix correspondent aux quartiles pour l'ensemble de la période 1998-2003. Distinguer deux périodes de durée équivalente autorise une comparaison des prix. Le type de bien immobilier retenu est la maison car c'est le plus représentatif du secteur ${ }^{6}$.

L'observation révèle que la part des biens de plus de 260000 euros s'est accrue au cours des dix dernières années, et ceci au détriment de ceux de moins de 145000 euros, qui représentaient au moins la moitié des ventes dans les deux tiers des communes des Yvelines entre 1998 et 2000. Au sud de la voie express, les biens les plus chers ont toujours été plus nombreux dans les ventes qu'au nord. Au nord, les maisons sont au départ moins coûteuses mais la hausse tend à rattraper les prix du sud. Si l'écart existe toujours, il a tendance à s'atténuer. À proximité de l'Eure-et-Loir, les prix sont moins élevés mais là encore le rattrapage est en cours avec davantage de ventes supérieures à 195000 euros. Cette constatation de la hausse des prix permet de mettre en évidence différents profils de communes.

Pour les terrains constructibles, l'étude a été menée à partir des prix au m². Certes l'exposition du terrain, sa situation, sa configuration jouent dans le prix mais à surface égale, les écarts sont moins grands que pour les maisons. Entre 1998 et 2000, la proximité de Paris explique pour l'essentiel la répartition des prix. Un noyau de communes situées à l'est et sur l'axe de la N12, a des prix moyens au $\mathrm{m}^{2}$ compris entre 96 et 171 euros. Autour de ce noyau, à l'intérieur d'un cercle passant à Orgerus les prix sont majoritairement compris entre 42 et 60 euros le $\mathrm{m}^{2}$. Au-delà, la gamme des prix est de l'ordre de 12 à 41 euros le $\mathrm{m}^{2}$, c'est-à-dire deux fois moins élevés qu'à l'est. Pour les terrains, le différentiel nord-sud n'existe pas, contrairement à ce qu'on a pu observer pour les maisons. Entre 2001 et 2003, le noyau des communes les plus cotées au départ garde les

6. Toutes les maisons sont prises en compte, quelle que soit leur taille, car la disparité en terme de nombre de pièces est équivalente sur l'ensemble des communes. 
prix les plus élevés tandis que les prix du cercle suivant amorcent un rapprochement en passant majoritairement dans la tranche supérieure, de 61 à 95 euros au $\mathrm{m}^{2}$. Il en est de même pour les prix des communes situées à l'ouest. Ils atteignent une fourchette de 42 à 60 euros au m², mais l'augmentation est moins forte puisque plusieurs communes situées aux limites de l'île-de-France conservent des prix inférieurs à 41 euros le $\mathrm{m}^{2}$.

Cette hausse des prix peut-elle être mise en relation avec l'aménagement de la voie express? Étant donné que ce mouvement concerne l'ensemble du territoire français, la difficulté est de distinguer ce qui relève d'une tendance générale et ce qui découle de la valorisation par la route.

Les observations faites au sujet de l'évolution contrastée des prix de l'immobilier et du foncier peuvent être synthétisées par la construction d'un indice d'évolution des prix entre 1998 et 2003. Le calcul consiste à rapporter l'indice d'évolution des prix dans la commune à celui calculé pour la France entière ${ }^{7}$. Quand il n'y a pas eu de transaction au cours de l'une des deux périodes, le calcul n'a pas été possible.

La carte figurant les évolutions des prix fonciers (fig. 3) distingue clairement les communes dont les prix ont grimpé plus vite que la moyenne française (aplat de couleur) des autres (figurées en hachures). Elle montre bien que les terrains à bâtir n’ont pas été insensibles à des paramètres locaux dans l'évolution de leurs prix. On retrouve l'ensemble de communes très cotées autour de Gambais où le niveau des prix était déjà élevé et qui ne font guère l'objet d'une pression foncière récente en rapport avec la voie express. Ces communes, proches du massif forestier de Rambouillet et de nombreux haras, ont toujours été des lieux de villégiature très prisés. Il en est de même pour des communes privilégiées situées à l'est, comme Neauphle-le-Vieux, Le-Tremblay-sur-Mauldre, Thiverval. En revanche la situation est différente pour des communes moins cotées. Prenons le cas de Plaisir pourtant située sur la N12 et proche de l'agglomération parisienne. La ville, où de nombreux ensembles collectifs accueillent des populations défavorisées, n’a guère une image positive, sans compter la présence sur son territoire de plusieurs zones d'activité. Plaisir connaît pourtant une pression importante. Cette commune fait jouer plusieurs atouts de proximité : l'axe N12, un nœud ferroviaire (connexion des deux lignes vers Mantes et vers Dreux) avec une liaison de Plaisir à Versailles en 15 minutes et à Paris-Montparnasse en 25 minutes par train direct. Partis de plus bas, les prix ont eu une marge de croissance plus forte. Le même cas de figure se produit pour des communes, éloignées de la N12, mais bien reliées à la voie express par des départementales. C'est là que s'exerce la pression foncière. Les prix y ont augmenté de 20 à $50 \%$ de plus qu'en moyenne française. En résumé, la pression foncière s'exerce sur les communes davantage en fonction de leur profil social avant l'aménagement de la voie express qu'en fonction de leur localisation par rapport à l'axe routier. Le passage de la voie ou la proximité de celle-ci ne joue qu'une fois la valorisation entamée, c'est-à-dire qu'elle ne fait que l'accentuer.

Le même calcul a été réalisé pour étudier l'évolution des prix de l'immobilier (fig. 4). L'indice d'évolution du prix médian des maisons corrigé de l'inflation moyenne observée pour la France entière offre des résultats différents. La pression immobilière apparaît bien atténuée par rapport à ce qu'on observe pour le foncier. Le plus souvent, les prix ont augmenté moins fortement qu'en moyenne française. Une grande partie des communes, situées le long de la N12, a des indices compris entre 90 et 99. Les prix sur ces communes se rapprochent donc de la tendance que connaît la France entière. Dans ce cas la route ne fait que sanctionner une évolution qui existe déjà : d'une part, une valorisation des communes les moins cotées situées au nord de la voie express, qui font figure de réserve immobilière et permettent une extension du marché; d'autre part, des communes déjà très cotées proches du massif de Rambouillet, situées au sud de l'axe routier. On ne peut donc

\footnotetext{
7. Les étapes intermédiaires sont les suivantes :

- (prix médian de la commune pour la période 2001-2003/prix médian de la commune pour la période 1998-2000) x 100 = indice d'évolution de la commune pour la période 2001-2003 par rapport à la base 100 (1998-2000).

- indice de la commune / indice calculé pour la France entière $(130,61)$ x $100=$ indice d'évolution des prix médians corrigé de l'inflation entre 1998 et 2003.
} 


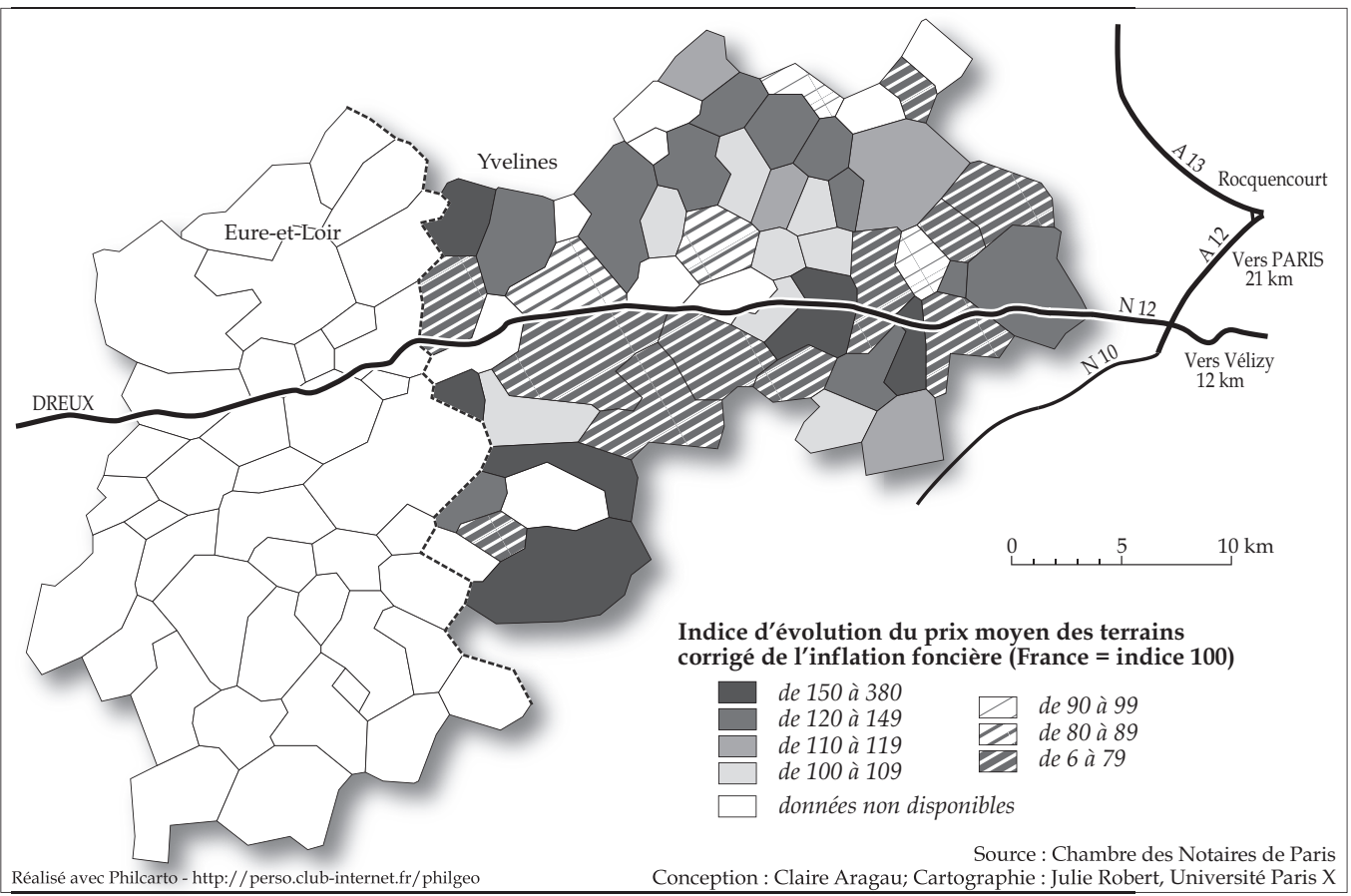

Figure 3 : Pression foncière et voie express : une réalité contrastée (évolution des prix entre 1998 et 2003)

Pressure on the land market and expressway : a contrasted fact (prices trend between 1998 and 2003)

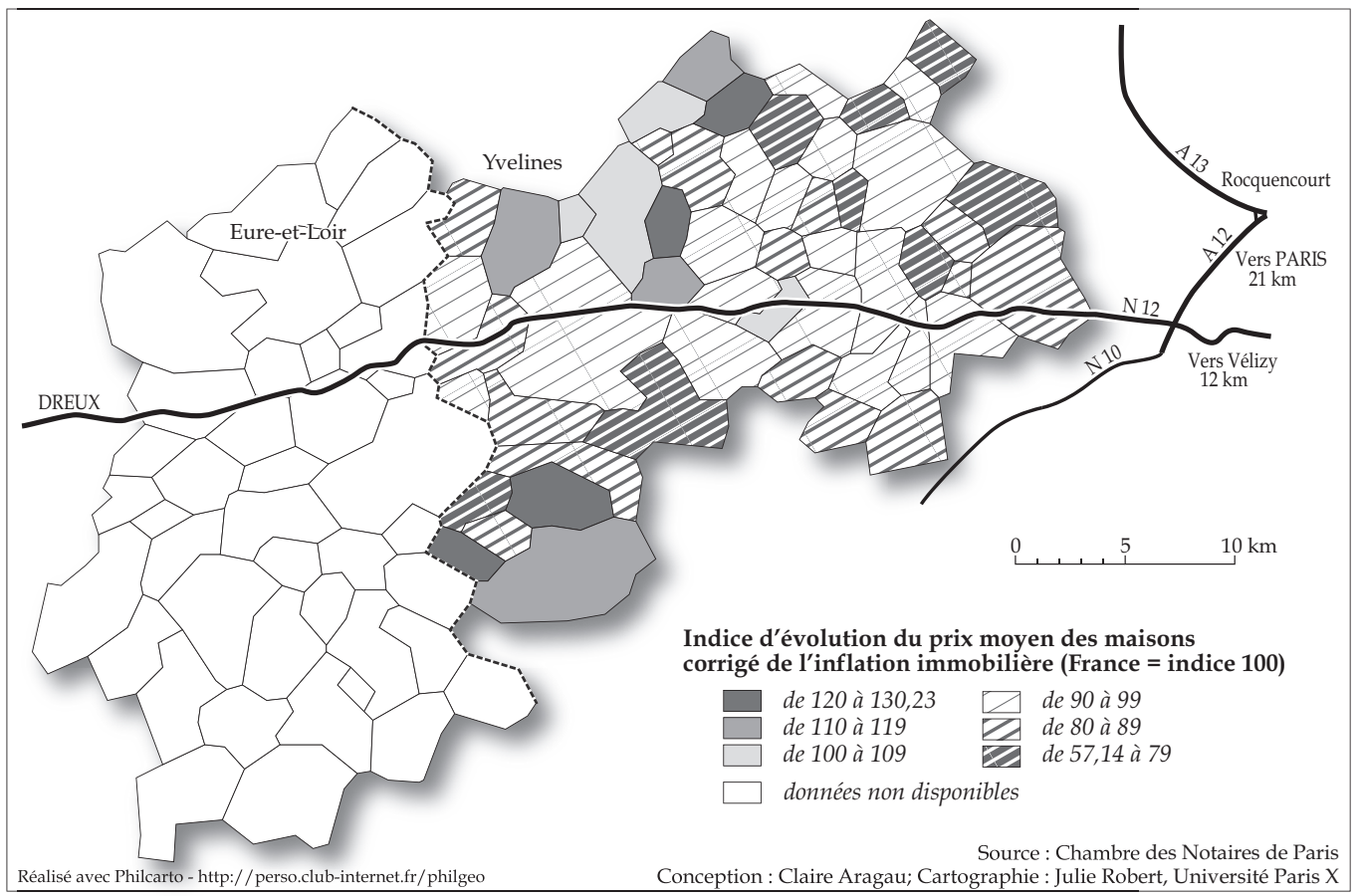

Figure 4 : Immobilier et voie express : une pression moins forte (évolution des prix entre 1998 et 2003) Property market and expressway: a slight pressure (prices trend between 1998 and 2003) 
pas parler franchement de pression immobilière, dans la mesure où le marché n'est pas encore saturé, et où la demande des ménages prêts à surenchérir, n'est pas encore supérieure à l'offre.

\section{LES DÉPLACEMENTS VERS L'OUEST}

D. Desponds (2006) évoque des logiques de «substitution sociale » lorsqu'il compare le profil socioprofessionnel des acquéreurs de biens immobiliers à celui des vendeurs dans le Val-d'Oise et le Parc Naturel Régional du Vexin français. Ce phénomène s'observe bien lorsque des particuliers, pour espérer revendre au meilleur prix, font jouer nettement la carte de la voie express. La stratégie qu'ils adoptent participe largement au processus de ségrégation sociale de l'espace repoussant vers l'ouest des ménages les plus modestes. Si l'on suit la N12, le gradient est-ouest est particulièrement net. Dans les communes situées à l'est, les cadres et professions libérales sont fortement représentés comparativement aux communes situées à l'ouest. Les communes localisées au nord et au sud de la voie express offrent également des profils bien distincts. Même si cette opposition nord/sud concerne des groupes socioprofessionnels différents dans les deux départements, on la retrouve bien dans les deux cas. Dans les Yvelines, les communes situées immédiatement au sud de la N12 concentrent les espaces de résidence de catégories souvent très privilégiées. On y enregistre une proportion importante de cadres et de professions libérales. Le phénomène est bien plus atténué au nord de la voie express, où ce profil ne caractérise qu'un nombre limité de communes, disposant à la fois d'une bonne accessibilité (Dupuy, 2006) et de paysages de qualité. En allant vers l'ouest, le contraste nord/sud est également visible même s’il s'atténue. À partir de Houdan, dans les Yvelines, les espaces résidentiels sont marqués par une forte présence des ménages d'employés. Au nord de la N12, la monotonie de la plaine s'oppose au paysage plus vallonné des bords de l'Eure au sud. Étangs et bosquets ont une valeur d'agrément supérieure. Les communes situées au sud, davantage marquées par la présence de professions libérales, recouvrent les principaux traits caractéristiques d'un espace favorisé.

Pour les communes d'Eure-et-Loir, on dispose d'informations issues de la base SITADEL ${ }^{8}$. Elles mettent en évidence les processus de ségrégation sociale à l'œuvre (fig. 5). Si l'on s'intéresse uniquement aux demandeurs de permis de construire originaires de la zone d'étude, on constate une forte dissymétrie au détriment des Yvelines. Les demandeurs issus de l'Eure-et-Loir et désirant faire construire dans les Yvelines sont bien peu nombreux par rapport aux Yvelinois s'installant dans des communes situées plus à l'ouest. La localisation de leur projet de construction laisse supposer qu'ils souhaitent profiter de l'amélioration des conditions de circulation offertes par la voie express. En effet les communes concernées par ces demandes se trouvent toutes sur la N12. Elles restent aussi majoritairement proches des limites de l'Eure-et-Loir, là où les prix leur sont certainement plus accessibles que dans les Yvelines (Sencébé, 2006). Les ménages d'Eure-et-Loir ont réalisé souvent une plus-value moins importante que ceux qui viennent des Yvelines; ils ont donc plus de mal à s'installer plus près de l'agglomération parisienne, dans la partie est de la zone d'étude. Durant la seconde période (1999-2003), le phénomène s'amplifie avec un nombre plus important de communes d'Eure-et-Loir concernées par l'arrivée de résidents yvelinois. De même, les originaires d'Eure-et-Loir sont moins nombreux à faire construire dans les Yvelines.

La déconnexion entre les prix fonciers et les prix immobiliers suscite une interrogation : la construction neuve, qui conduit à une forte demande en terrains à bâtir, n'est-elle pas préférée à l'habitat ancien? Les maisons existantes sont souvent considérées par les nouveaux résidents

8. La base SITADEL (Système d'information et de traitement automatisé des données élémentaires sur les logements et les locaux [www.statistiques.equipement.gouv.fr]) répertorie les permis de construire; elle remplace la base SICLONE à partir de 1994. Pour obtenir des données comparatives sur les dernières années, il faut donc s'en tenir à 1994 comme date de départ. Pour pouvoir avoir deux périodes de comparaison il est nécessaire de faire une césure entre 1998 et 1999 , sachant que 2003 est la dernière année dont nous disposons (La remontée des données aux services statistiques des Directions Régionales de l'Équipement est souvent longue). Cette base qui concerne les permis de construire délivre plusieurs informations dont le lieu de résidence du maître d'ouvrage. 


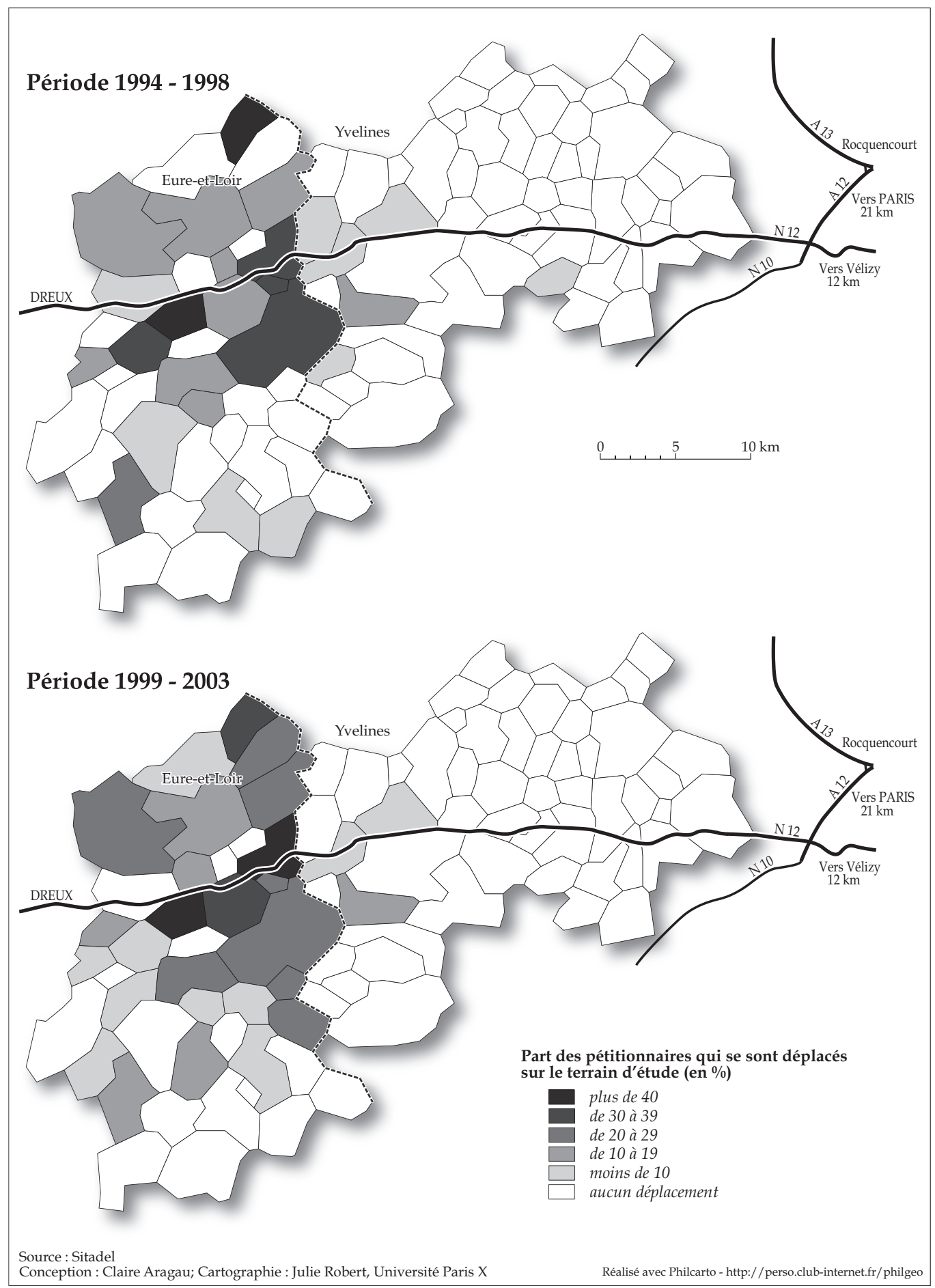

Figure 5 : Les pétitionnaires originaires du terrain d'étude Petitioners from studied area 
comme inadaptées au confort actuel, les demeures anciennes comme trop grandes (elles comptent parfois neuf pièces). Ce type de bien ne répond pas à la demande du marché qui tourne autour de 4-5 pièces. Si elles ne sont pas trop grandes, ces maisons sont trop petites avec des pièces sombres, mal adaptées aux exigences actuelles des acheteurs, telles que la présence de deux salles de bain. Outre le fait d'accéder à un habitat fonctionnel et répondant au confort moderne, c'est le coût final de l'opération qui fait préférer la construction neuve. En effet celui-ci est moins élevé que l'achat d'une maison existante. Les constructeurs passent des contrats avec des artisans pour obtenir des prix très compétitifs. C'est un argument de taille qui peut expliquer que l'on peut parler sur notre terrain d'étude davantage d'une pression foncière liée à la nouvelle N12 que d'une pression immobilière. M. Berger rappelle que si ce type de pavillon de plus en plus standardisé se répand, « il convient toutefois de réagir contre une vision trop égalitariste de la périurbanisation : s’il est vrai que nombreux sont ceux pour qui s'ouvre la perspective d'un accès à la propriété et/ou à la maison individuelle, ce n'est ni dans les mêmes lieux, ni avec le même niveau et la même durée d'endettement » (Berger, 2004). Pression foncière pour la construction neuve et renouvellement sélectif des profils des habitants jouent en interaction et de manière hétérogène sur le territoire étudié.

Le marché immobilier et foncier le long de la voie express n'offre pas une tendance homogène sur les communes proches de l'axe N12. Si la hausse des prix est nette, elle concerne avant tout les biens situés dans des communes peu valorisées au départ. Celles-ci se situent principalement au nord de la voie express par opposition à celles qui se trouvent au sud et qui sont adossées au massif forestier de Rambouillet. Deux éléments jouent en faveur d'une plus forte valorisation de certains espaces résidentiels. Il s'agit d'un accès rapide à la voie express qu'il soit plus direct ou qu'il se fasse par l'intermédiaire d'une bonne connexion au réseau secondaire. Ce critère vient se conjuguer à celui de la qualité paysagère du lieu. La proximité d'un cours d'eau, d'un bois ou une topographie vallonnée jouent en faveur d'un environnement considéré comme étant de qualité. Ces caractères paysagers sont perçus comme constituant un écran vert ou naturel pour se protéger de l'axe routier susceptible d'apporter des désagréments.

Parallèlement au désenclavement de certaines communes, le profil des résidents évolue. Les ménages des catégories modestes voire moyennes sont repoussés vers l'ouest ou vers des communes éloignées de l'axe routier central. Il est probable que le même phénomène se reproduira à l'avenir au-delà de Dreux, puisqu'il est question de poursuivre les aménagements de la N12 jusqu'à Verneuil-sur-Avre. Si l'on peut parler de ségrégation socio-spatiale, celle-ci s'opère à des rythmes différents, elle est fonction du profil social de la commune avant les aménagements de l'axe routier et de la capacité des élus à la doter des atouts actuellement recherchés : une bonne accessibilité et une bonne qualité de protection paysagère.

\section{Bibliographie}

Aragau (C.), 2007. - Aménagement d'une voie express et renouvellement périurbain: le cas de la N12, thèse de doctorat en géographie humaine, université de Paris 10 - Nanterre, 318 p.

Atlas rural et agricole de l'île-de-France, IAURIF, 2005, 180 p.

Berger (M.), 1993. - « Pour loger au large, logeons au loin! », Regards sur l'̂̂le-de-France, n 20, juin, p. 6-9. —, 2004. - Les périurbains de Paris. De la ville dense à la métropole éclatée?, Paris, CNRS, 317 p.

Biasi (L. de), Ordonnet (M.), 2004. - « La ruralité vue par les Franciliens : choix, pratiques et modes de vie », dans Biasi (L. de), Stephan (J.-M.), Mauclair (C.) (dir.), Atlas rural et agricole de l'île-de-France, Paris, Institut d'Aménagement et d'Urbanisme de la Région Île-de-France (IAURIF), p. 18-19.

Charmes (E.), 2005. - La vie périurbaine face à la menace des gated communities, Paris, L'Harmattan, $228 \mathrm{p}$. 
Desponds (D.), 2006. - « Spécialisations socio-résidentielles : vers des divergences territoriales accrues dans le cadre du Val-d'Oise? ", Hérodote, Ghettos américains, banlieues françaises, n 122-2006/3, p. 172-197.

Dubois-Taine (G.), Chalas (Y.), 1997. - La ville émergente, Éditions de l'Aube, 285 p.

Dupuy (G.), 2006. - La dépendance à l'égard de l'automobile, La Documentation française, PREDIT (Programme de recherche et d'innovation dans les transports terrestres), $96 \mathrm{p}$.

Jaillet (M.-C.), Rougé (L.), Thouzellier (C.), 2005. - «Vivre en maison individuelle en lotissement », dans TAPIE (G.) (dir.), Maison individuelle, architecture, urbanité, Éditions de l'Aube, p. 11-23.

Mouillart (M.), 2006. - « La crise du logement en France, pourquoi et pour qui ? », Regards sur l'actualité, $n^{\circ} 320$, avril 2006, p. 5-18.

OFFNER (J.-M.), 1993. - «"Les effets structurants" du transport : mythe politique, mystification scientifique », l'Espace géographique 1993-3, p. 233-242.

SEncÉbÉ (Y.), 2006. - « Mobilités quotidiennes et ancrages périurbains, attrait pour la campagne ou retrait de la ville? » dans Bonnet (M.), Aubertel (P.) (dir.), La ville aux limites de la mobilité, Paris, PUF, coll. « Sciences Sociales et Sociétés », p. 153-160.

Cet article a été reçu le le 4 octobre 2007 et définitivement accepté le 22 décembre 2007. 